\title{
Optimal claim behaviour for third-party liability insurances or To claim or not to claim: that is the question
}

\author{
N.P. DELLAERT, J.B.G. FRENK and A. KOUWENHOVEN \\ Econometric Institute, Erasmus University Rotterdam, 3000 DR Rotterdam, The Netherlands
}

B.S. VAN DER LAAN

Goudse Verzekeringen, Stationsplein 3, 2801 AK Gouda, The Netherlands

Received 4 June 1989

Revised 30 October 1989

It is proved that the optimal decision rule to claim or not to claim for damage is of the form: 'to claim for damage only if its amount exceeds a certain limit'. Optimal critical claim sizes are derived, and a sensitivity analysis is given with respect to changes in (the parameters of) the distributions of the number of claims and the claim size.

Keywords: Optimal critical claim size, Markov process, Sensitivity analysis.

\section{Introduction}

An important feature of premium rating systems for vehicle insurance is the no-claim or bonus-malus principle. This principle is meant to reward policy holders for not making claims during a year; that is, to grant a bonus to a careful driver. A bonus principle effects the policy holder's decision whether or not to claim in a particular instance. No claim will be made for some of the accidents where there is only slight damage. Philipson (1960) called this phenomenon 'hunger for bonus'.

Little is known about the real behaviour of policy holders with respect to the no-claim principle. It can, however, be expected that an individual policy holder will only claim for damage when its amount exceeds a certain limit, which is assumed by most authors. We may assume that the higher the number of claim-free years or the higher the class of the bonus-malus principle, the higher this limit will be. The policy holder must realize that

- if he claims for damage, the insurance company will pay for the damage, but he will pay a higher premium for a number of years;

- if he does not claim, he has to pay for the damage himself, but the premium he must pay for the next insurance year does not increase.

The premium for insuring a vehicle must be paid at the beginning of an insurance year. The amount of the premium depends on the bonus-malus class the policy holder at present belongs to. At the end of each year the policy holder moves to another class, according to certain rules, depending only on the fact whether he filed any claim during that year. We notice that the amount of the claim does not matter, only the number of claims determines the bonus-malus class to which the policy holder will belong during the next year. An example of a bonus-malus scheme operative in the Netherlands is given in Table 1.

In this paper we restrict our analysis to a third-party liability insurance, where the total amount of damage is covered, i.e., there is no deductible. The analysis is based on the definition of a state presented 
Table 1

Percentages of the basic premium by bonus-malus class.

\begin{tabular}{|c|c|c|c|c|c|}
\hline \multirow{2}{*}{$\begin{array}{l}\text { Bonus-malus } \\
\text { class }\end{array}$} & \multirow{2}{*}{$\begin{array}{l}\text { Premium as of the } \\
\text { basic premium }\end{array}$} & \multicolumn{4}{|c|}{ New bonus-malus class after ... claims } \\
\hline & & $\overline{0}$ & 1 & 2 & $\geq 3$ \\
\hline 14 & 30.0 & 14 & 9 & 5 & 1 \\
\hline 13 & 32.5 & 14 & 8 & 4 & 1 \\
\hline 12 & 35.0 & 13 & 8 & 4 & 1 \\
\hline 11 & 37.5 & 12 & 7 & 3 & 1 \\
\hline 10 & 40.0 & 11 & 7 & 2 & 1 \\
\hline 9 & 45.0 & 10 & 6 & 1 & 1 \\
\hline 8 & 50.0 & 9 & 5 & 1 & 1 \\
\hline 7 & 55.0 & 8 & 4 & 1 & 1 \\
\hline 6 & 60.0 & 7 & 3 & 1 & 1 \\
\hline 5 & 70.0 & 6 & 2 & 1 & 1 \\
\hline 4 & 80.0 & 5 & 1 & 1 & 1 \\
\hline 3 & 90.0 & 4 & 1 & 1 & 1 \\
\hline 2 & 100.0 & 3 & 1 & 1 & 1 \\
\hline 1 & 120.0 & 2 & 1 & 1 & 1 \\
\hline
\end{tabular}

by Norman and Shearn (1980). So we assume a discrete time axis. This assumption is not very restrictive in practical situations, for a policy holder is generally allowed some time (at least 24 hours) to decide to claim or not to claim for damage. ${ }^{1}$ We will prove that - given some assumptions - the optimal decision rule to claim or not to claim, in such a way that the expected discounted costs are minimal, is of the form: 'to claim for damage only if its amount exceeds a certain limit'. We derive a system of recurrent equations, the solution of which gives the optimal critical claim sizes. These critical claim sizes are derived for both the case of a finite and that of an infinite horizon. It will appear that the optimal critical claim size depends on the probability distributions of the number of claims and the claim size. Therefore, we also analyse the sensitivity of the optimal critical claim size with respect to changes in (the values of the parameters of) the applied probability distributions. We finish our analysis with an example based on a Dutch bonus-malus premium rating system.

\section{Review of the literature}

No-claim problems have been discussed since the fifties. Several ASTIN meetings paid attention to this subject. Derron (1965) states "that a subsequent adjustment of premiums according to the past claim record may well be a suitable way of obtaining a fair premium'. Gürtler $(1960,1961,1962)$ introduced a standard for evaluating the fairness of a premium. Derron (1965) extends and complements the results obtained by Gürtler.

Welten (1969) points out that the bonus a policy holder obtains usually consists of at least three components which depend on the length of time preceding the current insurance period: a component concerning the individual claim frequency, an individual random factor and a collective random factor. The last two components tend to zero for increasing length of time. The sum of these last two components, called 'unearned bonus', should be taken into account by insurance companies in the short run, and would lead to a bonus reserve.

Alting von Geusau (1968) investigates 'to what extent it is possible to develop a theoretical framework to test $(\ldots)$ that a no-claim-discount-system will prevent the insured from submitting small claims to the

1 Recently, some Dutch insurance companies allow an insurant to decide at the end of the insurance year whether he will file all claims made during that year or to take for his own account the costs of one or more claims. 
insurance company', and 'that the insured who has just lost his no-claim discount will use every possibility for submitting claims with in his mind the idea that in this way he will earn back his higher non-reduced premium'.

Loimaranta (1972) develops formulas for some asymptotic properties of bonus systems, where bonus systems are understood as Markov chains. He introduces the quantities: efficiency of a bonus system, discriminatory power of bonus rules and minimum variance bonus scale. The last one gives an asymptotic solution for the problem to find locally 'best' bonus scales for given bonus rules. Bonus systems used in Denmark, Norway, Sweden, Finland, Switzerland and West Germany are studied by Vepsäläinen (1972), on the basis of the method given by Loimaranta. Lemaire (1976) defines an efficiency concept for a bonus-malus system, which differs from the concept given by Loimaranta (1972). De Pril (1978) presents a more general concept of efficiency, which includes both earlier ones as special cases.

Grenander (1957) derives equations to determine a rule of the form "pay the damage if its amount is smaller than a critical value and claim it otherwise'. However, the equations are generally difficult to solve, and it is not proved that they really determine an optimal policy in the sense that the total expected discounted cost of premiums and payments during a long future planning period is minimized.

Haehling von Lanzenauer $(1969,1972 a, b)$ analyses the problem on the assumption that a policy holder can cause at most one accident per year.

De Leve and Weeda (1968) develop a mathematical model, called generalized Markov programming, that yields an optimal strategy, which is a function $s(t)$ that minimizes the expected costs for the policy holder. This function $s(t)$ is such that "if at any time $t$ an accident occurs with damage $s$ and no damages have been claimed since the last payment of premium, then $s$ should be claimed if $s>s(t)$ '. In this approach the decision depends on the point of time during the year and the premium paid at the beginning of that year. De Leve and Weeda allow more than one accident per year, hut restrict their analysis to the case where, after making one claim during an insurance period, the policy holder is placed in the class with the highest premium. Weeda (1975) extends the analysis of the same model to the case where the damage distribution is given by an arbitrary distribution and focuses on the theoretical aspects of the derived iteration scheme. However, although the model is continuous with respect to the time axis, he discretizes time for computational purposes.

Martin-Löf (1973) shows that a decision rule of the form formulated by Grenander is optimal in the sense that it minimizes the total expected costs. The decision rule is derived by applying the general theory of Markov decision processes, which uses dynamic programming to find an optimal control iteratively. Martin-Löf, however, restricts the analysis to the case where the policy holder takes a decision only at the end of an insurance period for the total amount of damage sustained during that insurance period.

Haehling von Lanzenauer and Lundberg (1973) develop a model which can be used in deriving the distribution of the number of claims for insurances with merit-rating structures. The problem is formulated and solved as a regular Markov process with the claim behaviour integrated in the analysis.

Haehling von Lanzenauer (1974) develops an optimal decision rule - for situations where the policy holder takes a decision more than once a year - which is valid for any merit-rating system. He splits up a year into a number of periods, which results in a discrete model in which the optimal critical claim size can be determined by dynamic programming. However, his derivation of an optimal critical claim size is obscure.

Lemaire (1977) derives an algorithm for obtaining the optimal strategy for a policy holder. In his model the policy holder remains always insured (the so-called infinite horizon model) which leads to a critical claim size which is independent of the year in which the accident takes place. Also, in order to compute the optimal policy, he uses policy iteration, which is very time-consuming, whenever the state space is large. Lemaire (1976) applics this algorithm to compare bonus systems used in Denmark, Norway, Sweden, Finland, Switzerland and West Germany.

Hastings (1976) presents a simple model based on a typical British policy, assuming that the number of accidents is Poisson and the amount of damage is negative exponentially distributed. He assumes an optimal critical claim size, which is constant throughout the year, irrespective of the number of claims already made during the year, and irrespective of the time until the next premium payment. He determines 
optimal critical claim sizes, which minimize the long-run average costs of premiums and repairs. The problem is formulated as a Markov decision problem and is solved by dynamic programming.

Almost all studies mentioned above have in common that they assume a discrete time axis. De Pril (1979) gives a formulation based on a continuous time axis, where the optimal critical claim size can be determined by solving a set of recurrent differential equations. However, for solving these equations, a discretization is needed, giving rise to the same results as in Haehling von Lanzenauer (1974). Norman and Shearn (1980) build on Hastings' model, where they drop the restriction of a constant optimal critical claim size. Moreover, they present a much simpler state description than the one used by Haehling von Lanzenauer (1974). The optimal decision rule has been compared with rules of thumb that appear to produce remarkably good results. Tijms (1986, pp. 196-200) gives a model that is equal to that presented by Norman and Shearn as an illustration.

Kolderman and Volgenant (1985) present a continuous model based on generalized Markov programming, applicable to bonus-malus systems used by Dutch motor insurance companies. However, in the computational part of their study they discretize time for numerical reasons.

Lemaire (1985, Chapter 18) describes a simple model with the assumption that all claims are reported in the middle of the insurance period.

Menist and Volgenant (1986) compute the optimal critical claim size by considering the difference between the expected costs in case of claiming and that of not claiming damage. They restrict the analysis to a finite horizon.

\section{The model}

In this section we derive a discrete model for the decision problem to claim or not to claim damage. Therefore, we need three spaces: the state space, the decision space and the set of decision moments.

We divide an insurance period, generally a year, in $N$ equal periods. We assume that the decision to claim or not to claim damage has to be taken at the end of a period. Let the decision process continue $T$ years $(T \leq \infty)$ and start at the beginning of a certain year. Then the set of decision moments is defined as

$$
\mathscr{T}:=\{\tau=1,2, \ldots, H\},
$$

where $H=N T$.

Next, we consider the probability distributions of the number of accidents and the amount of damage. We assume that the size of $N$, the number of periods in which the total insurance year is divided, is such that the probability of two or more accidents in any period is negligible. Further, we assume that the probability of one accident in a certain period varies from one period to another. We define the probability

$$
p_{n}=\operatorname{Pr}\left(' 1 \text { accident in period } n^{\prime}\right), \quad n=1, \ldots, N,
$$

and a random variable $\underline{Z}_{n}$, where

$$
\underline{Z}_{n}=i(i=0,1) \text { if } i \text { accidents occur in period } n \text {. }
$$

Then we have

$$
\operatorname{Pr}\left[\underline{Z}_{n}=1\right]=p_{n} \quad \text { and } \operatorname{Pr}\left[\underline{Z}_{n}=0\right]=1-p_{n} .
$$

We assume $\underline{Z}_{n}(n=1, \ldots, N)$ to be mutually stochastically independent.

Accidents generally imply damage. We define the random variable $\underline{Y}_{n}$ as the amount of damage resulting from an accident in period $n$. We assume $Y_{n}(n=1, \ldots, N)$ to be stochastically independently distributed. The $Y_{n}$ 's have the same probability distribution, but the parameters of their probability distributions depend on the period in which the accident occurs. Let $F_{n}(\cdot)$ and $f_{n}(\cdot)$ be the probability distribution function and the probability density function of $\underline{Y}_{n}$. Finally, we assume that the damage resulting from any accident is positive, so

$$
f_{n}\left(y_{n}\right)=0, \quad y_{n} \leq 0, n=1, \ldots, N
$$


Finally, if we denote the total amount of damage in period $n$ by the random variable $\underline{X}_{n}$, then clearly

$$
\begin{aligned}
\underline{X}_{n} & =\underline{Y}_{n} & \text { if } & \underline{Z}_{n}=1, \\
& =0 & \text { if } & \underline{Z}_{n}=0 .
\end{aligned}
$$

Hence the probability distribution function $G_{n}(\cdot)$ belonging to the random variable $\underline{X}_{n}$ equals

$$
\begin{aligned}
G_{n}(x) & =\operatorname{Pr}\left[\underline{X}_{n} \leq x \mid \underline{Z}_{n}=0\right] \operatorname{Pr}\left[\underline{Z}_{n}=0\right]+\operatorname{Pr}\left[\underline{X}_{n} \leq x \mid Z_{n}=1\right] \operatorname{Pr}\left[\underline{Z}_{n}=1\right] \\
& =1-p_{n}+p_{n} F_{n}(x)
\end{aligned}
$$

for every $x \geq 0$.

Moreover, the corresponding probability density function $g_{n}(\cdot)$ is given by

$$
\begin{array}{rlrl}
g_{n}(x) & =1-p_{n} & \text { if } & x=0, \\
& =p_{n} f_{n}(x) \text { if } & x>0 .
\end{array}
$$

When a policy holder is involved in an accident during a certain period, he has to decide to claim or not to claim the resulting damage. Consequently, the decision space is defined as

$$
\mathscr{B}:=\{b \mid b=0,1\},
$$

where 1 denotes to claim and 0 denotes not to claim. It is self-evident that when no accident occurs during a certain period, the policy holder does not claim at the end of that period. Finally, we must define a state $E_{\gamma}$ at each decision moment. Haehling von Lanzenauer (1974) defines the state space on the basis of four components:

$i=$ the policy holder's bonus-malus class,

$k=$ the number of claims already filed during the current year,

$n=$ the index defining a period,

$x=$ the amount of damage resulting from an accident occurring in period $n$, where $x=0$ when no accident occurs.

A state is then given by

$$
E_{\gamma}=(i, k, n, x)
$$

and the state space is defined as the set of states $E_{\gamma}$. Norman and Shearn (1980) simplify the state to three components:

$$
E_{\gamma}:=(j, n, x),
$$

where the components $n$ and $x$ are defined as before. The component $j$ is determined by

(1) the premium to be paid next year by the policy holder when he does not claim damage during the rest of the current year $\left(\pi_{0}\right)$,

(2) the premium to be paid next year by the policy holder when he claims damage exactly once during the rest of the current year $\left(\pi_{1}\right)$,

As will appear below, the premium, that he would pay when he would claim two or more times during the rest of the current year, does not play any role.

We can elicit every possible combination of $\pi_{0}$ and $\pi_{1}$ from the transition table of the bonus-malus scheme; let there be $J$ such combinations. We denote a particular combination with $j$, where $j=1, \ldots, J$.

Now we consider the two cases where $j$ changes.

(1) The policy holder files a claim. The combination $\left(\pi_{0}, \pi_{1}\right)$ does not change, when the policy holder does not file a claim. When he files a claim, he moves to a new combination $\left(\pi_{0}^{*}, \pi_{1}^{*}\right)$, where $\pi_{0}^{*}=\pi_{1}$. We define $a(j)$ as the number of the new combination when the policy holder files a claim, and when the 
Table 2

Norman and Shearn's definition of the state space applied to Table $1 .^{a}$

\begin{tabular}{|c|c|c|c|c|}
\hline$j$ & $\rho_{0}(j)$ & $\rho_{1}(j)$ & $a(j)$ & $b(j)$ \\
\hline 1 & 120.0 & 120.0 & 1 & 2 \\
\hline 2 & 100.0 & 120.0 & 1 & 3 \\
\hline 3 & 90.0 & 120.0 & 1 & 4 \\
\hline 4 & 80.0 & 120.0 & 1 & 5 \\
\hline 5 & 70.0 & 120.0 & 1 & 7 \\
\hline 6 & 60.0 & 120.0 & 1 & 9 \\
\hline 7 & 60.0 & 100.0 & 2 & 9 \\
\hline 8 & 55.0 & 100.0 & 2 & 10 \\
\hline 9 & 55.0 & 90.0 & 3 & 10 \\
\hline 10 & 50.0 & 80.0 & 4 & 11 \\
\hline 11 & 45.0 & 70.0 & 5 & 12 \\
\hline 12 & 40.0 & 60.0 & 6 & 13 \\
\hline 13 & 37.5 & 55.0 & 8 & 14 \\
\hline 14 & 35.0 & 55.0 & 9 & 15 \\
\hline 15 & 32.5 & 50.0 & 10 & 16 \\
\hline 16 & 30.0 & 50.0 & 10 & 17 \\
\hline 17 & 30.0 & 45.0 & 11 & 17 \\
\hline
\end{tabular}

The functions $\rho_{0}(j)$ and $\rho_{1}(j)$ equal $\pi_{0}(j)$ and $\pi_{1}(j)$ as a percentage of the basic premium.

previous combination has number $j$. This can be represented schematically by

$$
j \rightarrow\left\{\begin{array}{ll}
j & \text { no claims } \\
a(j) & \text { one claim }
\end{array} .\right.
$$

It follows that it is not necessary to keep how many claims per year are filed, because the next year premium is adapted immediately after a claim is filed.

(2) A new insurance year takes effect. The policy holder pays premium at the beginning of each insurance year. This premium is equal to $\pi_{0}(a(j))$ or $\pi_{0}(j)$, depending on whether he has or has not filed a claim in state $(j, N, x)$. When he does not file a claim during a year, he moves to a higher bonus-malus class (unless he is already in the highest class), and he will pay a lower premium next year than he did in the current year. This corresponds to a different combination $\left(\pi_{0}, \pi_{1}\right)$. Consequently, concerning $j$ there are two transitions between the points of time $n=N$ of any year and $n=1$ of the next year:

$$
\begin{cases}j \rightarrow j \rightarrow b(j) & \text { when no claim is filed, } \\ j \rightarrow a(j) \rightarrow b(a(j)) & \text { when a claim is filed, }\end{cases}
$$

where $b(j)$ is defined as the number of the new combination as the result of the transition to the new year. Given a transition mechanism, as presented in Table 1, we can determine the values of $j, a(j), b(j)$ and of the corresponding premiums. Table 2 contains these values. We notice that different values will result, when a different bonus-malus system is effective.

The state space as formulated by Norman and Shearn contains less components and has less elements than the state space defined by Haehling von Lanzenauer, but is otherwise identical. The number of elements in the state space is important when the set of functional equations must be solved. Therefore, we prefer the state space definition of Norman and Shearn.

Finally, we must define the transition probabilities. That is, we must determine the probability density function corresponding to the state $E_{\delta}$, given that the policy holder was in state $E_{\gamma}$ at the previous point of time and has taken decision $b$. This function is denoted by $h\left(E_{\delta} \mid E_{\gamma}, b\right)$. Furthermore, we define $C\left(E_{\gamma}, b\right)$ as the costs in the next period, when decision $b$ has been taken in the current period and the policy holder is in state $E_{\gamma}$. Table 3 contains the values of these functions. 
Table 3

$C\left(E_{\gamma}, b\right)$ and $h\left(E_{\delta} \mid E_{\gamma}, b\right)$ for all $E_{\gamma}, E_{\delta}, b$ and $n$

\begin{tabular}{llllll}
\hline$E_{\gamma}$ & $n$ & $b$ & $E_{\delta}$ & $C\left(E_{\gamma}, b\right)$ & $h\left(E_{\delta} \mid E_{\gamma}, b\right)$ \\
\hline$(j, n, x)$ & $1, \ldots, N-1$ & 0 & $(j, n+1, y)$ & $x$ & $g_{n+1}(y)$ \\
& & 1 & $(a(j), n+1, y)$ & 0 & $g_{n+1}(y)$ \\
$(j, n, x)$ & $N$ & 0 & $(b(j), 1, y)$ & $x+\pi_{0}(j)$ & $g_{1}(y)$ \\
& & 1 & $(b(a(j)), 1, y)$ & $\pi_{0}(a(j))$ & $g_{1}(y)$ \\
\hline
\end{tabular}

For the other states the transition probabilities equal zero.

Given the definitions presented above, we are now able to formulate the above decision problems as Markov decision processes. ${ }^{2}$

Starting with a discounted finite model with an horizon of $N T$ periods, we introduce, for every $1 \leq i \leq T<\infty$ and $1 \leq n \leq N$,

$V_{i}(j, n, x):=$ minimal expected total discounted costs from period $N(T-i)+n$ through period $N T$ when the policy holder is in state $(j, n, x)$ at the end of period $N(T-i)+n$.

Obviously, the policy holder files all claims during the last year of the insurance. Therefore, for every state $(j, n, x), 1 \leq n \leq N$, we get

$$
V_{1}(j, n, x)=0 \text {. }
$$

Moreover, by the specific form of the one-period cost functions (cf. Table 3) and Bellman's principle of optimality [cf. Bertsekas (1976), Ross (1983)] we obtain the following set of backward equations:

For every $2 \leq i \leq T, 1 \leq n \leq N-1$ and $x>0$ we have

$$
V_{i}(j, n, x)=\min \left\{\begin{array}{l}
x+\beta \mathbb{E}\left(V_{i}\left(j, n+1, \underline{X}_{n+1}\right)\right) \\
\beta \mathbb{E}\left(V_{i}\left(a(j), n+1, \underline{X}_{n+1}\right)\right)
\end{array} .\right.
$$

For every $2 \leq i \leq T, 1 \leq n \leq N-1$ and $x=0$ we get

$$
V_{i}(j, n, 0)=\beta \mathbb{E}\left(V_{i}\left(j, n+1, \underline{X}_{n+1}\right)\right) .
$$

For every $2 \leq i \leq T, n=N$ and $x>0$ it holds that

$$
V_{i}(j, N, x)=\min \left\{\begin{array}{l}
x+\pi_{0}(j)+\beta \mathbb{E}\left(V_{i-1}\left(b(j), 1, \underline{X}_{1}\right)\right) \\
\pi_{0}(a(j))+\beta \mathbb{E}\left(V_{i-1}\left(b(a(j)), 1, \underline{X}_{1}\right)\right)
\end{array} .\right.
$$

Finally, for every $2 \leq i \leq T, n=N$ and $x=0$ we have

$$
V_{i}(j, N, 0)=\pi_{0}(j)+\beta \mathbb{E}\left(V_{i-1}\left(b(j), 1, \underline{X}_{1}\right)\right),
$$

where $\mathbb{E}$ denotes the mathematical expectation.

In order to simplify expressions we define, for every $1 \leq i \leq T$,

$$
\begin{aligned}
& V_{i}(j, n):=\mathbb{E}\left(V_{i}\left(j, n, \underline{X}_{n}\right)\right), \quad 1 \leq n \leq N, \\
& D_{i}(j, n):=\beta\left(V_{i}(a(j), n+1)-V_{i}(j, n+1)\right), \quad 1 \leq n \leq N-1,
\end{aligned}
$$

and, for every $2 \leq i \leq T$,

$$
D_{i}(j, N):=\beta\left(V_{i-1}(b(a(j)), 1)-V_{i-1}(b(j), 1)\right)+\pi_{0}(a(j))-\pi_{0}(j) .
$$

Clearly, $V_{i}(j, n)$ and $D_{i}(j, n)$ are non-negative for every $(j, n)$.

\footnotetext{
${ }^{2}$ For an overview on the theory of Markov decision processes the interested reader is referred to Van der Wal and Wessels (1985).
} 
By (2), (3), (4) and (5) we observe, for every $2 \leq i \leq T$,

$$
V_{i}(j, n, x)=\left\{\begin{array}{ll}
x+\beta V_{i}(j, n+1) & \text { if } \quad 0 \leq x \leq D_{i}(j, n) \\
\beta V_{i}(a(j), n+1) & \text { if } \quad x>D_{i}(j, n)
\end{array} \quad \text { whenever } \quad 1 \leq n \leq N-1,\right.
$$

and

$$
V_{i}(j, N, x)=\left\{\begin{array}{ll}
x+\pi_{0}(j)+\beta V_{i-1}(b(j), 1) & \text { if } \quad x \leq D_{i}(j, N) \\
\pi_{0}(a(j))+\beta V_{i-1}(b(a(j)), 1) & \text { if } \quad x>D_{i}(j, N)
\end{array} \text { for } n=N .\right.
$$

From (1), (9) and (10) it is obvious that the policy holder, observing state $(j, n, x)$ at the end of period $N(T-i)+n, 1 \leq i \leq T, 1 \leq n \leq N$, will claim if and only if the amount of damage exceeds a certain limit. After having identified the form of the optimal policy we are interested in the computation of this so-called threshold policy, i.e., the computation of the optimal critical claim sizes.

Clearly by (1) the optimal critical claim sizes at the end of period $n+N(T-1), 1 \leq n \leq N$ are zero. For the computation of the other critical claim sizes we need the equations (9) and (10).

By these relations we immediately obtain, for every $2 \leq i \leq T$,

$$
\begin{aligned}
V_{i}(j, n):= & \mathbb{E}\left(V_{i}\left(j, n, \underline{X}_{n}\right)\right) \\
= & \left\{\begin{array}{l}
K_{n}\left(D_{i}(j, n)\right)+\beta\left(V_{i}(j, n+1) G_{n}\left(D_{i}(j, n)\right)+V_{i}(a(j), n+1)\left(1-G_{n}\left(D_{i}(j, n)\right)\right)\right) \\
\quad \text { if } 1 \leq n \leq N-1, \\
K_{N}\left(D_{i}(j, N)\right)+\pi_{0}(j) G_{N}\left(D_{i}(j, N)\right)+\pi_{0}(a(j))\left(1-G_{N}\left(D_{i}(j, N)\right)\right) \\
+\beta\left(V_{i-1}(b(j), 1) G_{N}\left(D_{i}(j, N)\right)+V_{i-1}(b(a(j)), 1)\left(1-G_{N}\left(D_{i}(j, N)\right)\right)\right) \\
\quad \text { if } n=N,
\end{array}\right.
\end{aligned}
$$

where $K_{n}(d)$ is the expected amount of damage to be paid by the policy holder having critical claim size $d$ during period $n$;

$$
K_{n}(d):=\mathbb{E}\left(\underline{X}_{n} 1_{\left\{\underline{X}_{n} \leq d\right\}}\right)=p_{n} \int_{0}^{d} x \mathrm{~d} F_{n}(x), \quad 1 \leq n \leq N,
$$

and

$$
G_{n}(d):=P\left\{\underline{X}_{n} \leq d\right\}=\left\{\begin{array}{lll}
1-p_{n} & \text { if } & d=0 \\
1-p_{n}+p_{n} F_{n}(d) & \text { if } & d>0
\end{array} .\right.
$$

By (11) and (12) the optimal critical claim sizes can easily be found in the following recurrent way.

Clearly by (1) and (8) it holds that

$$
D_{2}(j, N)=\pi_{0}(a(j))-\pi_{0}(j) \text { for every } j .
$$

Hence by (12), $V_{2}(j, N)$ is known for every $j$ and this yields by (7) the value of $D_{2}(j, N-1)$ for every $j$. Suppose now we have computed for some $2 \leq i \leq T$ and $1 \leq n \leq N$ the values of $D_{2}(j, N)$, $V_{2}(j, N), \ldots, D_{i}(j, n)$ for every $j$.

By (11) (if $1 \leq n \leq N-1$ ) or (12) (if $n=N$ ) this yields $V_{i}(j, n)$ and hence by (7) and (8) $D_{i}(j, n-1)$ (if $n \geq 2$ ) or $D_{i+1}(j, N)$ (if $n=1$ ) is known.

The above iterative procedure for computing every critical claim size needs $N J(T-1)$ recurrent steps involving $O(1)$ computations and this implies that the complexity of the above algorithm is $O(N J T)$, assuming it takes a constant time to evaluate the functions in (11) or (12). This concludes for the time being our discussion of the finite $N T$-horizon model. In the next section we will discuss some sensitivity results for this model with respect to variations in the input parameters $F_{n}$ and $p_{n}, n=1, \ldots, N$. The remainder of this section is devoted to the analysis of the infinite horizon model.

Define

$V(j, n, x):=$ minimal expected total discounted costs if the policy holder observes state $(j, n, x)$ at the end of period $n$. 
As for the discounted finite horizon model we obtain the following set of recurrence relations.

For every $n=1, \ldots, N-1$ and $x>0$ we get

$$
V(j, n, x)=\min \left\{\begin{array}{l}
x+\beta \mathbb{E}\left(V\left(j, n+1, \underline{X}_{n+1}\right)\right) \\
\beta \mathbb{E}\left(V\left(a(j), n+1, \underline{X}_{n+1}\right)\right)
\end{array} .\right.
$$

For every $n=1, \ldots, N-1$ and $x=0$ we obtain

$$
V(j, n, 0)=\beta \mathbb{E}\left(V\left(j, n+1, \underline{X}_{n+1}\right)\right) .
$$

For every $n=N$ and $x>0$ it holds that

$$
V(j, n, x)=\min \left\{\begin{array}{l}
x+\pi_{0}(j)+\beta \mathbb{E}\left(V\left(b(j), 1, \underline{X}_{1}\right)\right) \\
\pi_{0}(a(j))+\beta \mathbb{E}\left(V\left(b(a(j)), 1, \underline{X}_{1}\right)\right)
\end{array} .\right.
$$

For $n=N$ and $x=0$ it yields

$$
V(j, N, 0)=\pi_{0}(j)+\beta \mathbb{E}\left(V\left(b(j), 1, \underline{X}_{1}\right)\right) .
$$

Similarly as for the finite horizon model we obtain that a policy holder, observing $(j, n, x)$ at some decision point, will claim if and only if $x>D(j, n)$, where

$$
\begin{aligned}
& V(j, n):=\mathbb{E}\left(V\left(j, n, \underline{X}_{n}\right)\right), \\
& D(j, n):=\beta(V(a(j), n+1)-V(j, n+1)) \quad \text { if } \quad 1 \leq n \leq N-1
\end{aligned}
$$

and

$$
D(j, N):=\pi_{0}(a(j))-\pi_{0}(j)+\beta(V(b(a(j)), 1)-V(b(j), 1)) .
$$

Clearly $D(j, n)$ and $V(j, n)$ are non-negative for every $(j, n)$.

Notice that the optimal policy for the discounted infinite horizon model is stationary as follows directly from the theory of Markov decision processes [cf. Ross (1983)].

In order to compute this threshold policy, i.e., the optimal critical claim sizes, we can proceed in the same way as for the finite horizon model. This yields the following set of equations:

$$
\begin{aligned}
V(j, n): & =\mathbb{E}\left(V\left(j, n, \underline{X}_{n}\right)\right) \\
& =\left\{\begin{array}{r}
K_{n}(D(j, n))+\beta\left(V(j, n+1) G_{n}(D(j, n))+V(a(j), n+1)\left(1-G_{n}(D(j, n))\right)\right) \\
\text { if } 1 \leq n \leq N-1,(20) \\
K_{N}(D(j, N))+\pi_{0}(j) G_{n}(D(j, N))+\pi_{0}(a(j))\left(1-G_{N}(D(j, N))\right) \\
+\beta\left(V(b(j), 1) G_{N}(D(j, N)) \mid V(b(a(j)), 1)\left(1-G_{N}(D(j, N))\right)\right) \\
\text { if } n=N .
\end{array}\right.
\end{aligned}
$$

The above system of equations can be solved by the well-known method of successive approximations [cf. Ross (1983)]. However, before proving this we have to rewrite (20) and (21) in a suitable form.

First of all, we apply Lemma A.1 (see the appendix) to these equations. This yields ${ }^{3}$

$$
\begin{aligned}
& V(j, n):=\mathbb{E}\left(V\left(j, n, \underline{X}_{n}\right)\right) \\
& = \begin{cases}\min _{d \geq 0}\left[K_{n}(d)+\beta\left(V(j, n+1) G_{n}(d)+V(a(j), n+1)\left(1-G_{n}(d)\right)\right)\right] \\
\min _{d \geq 0}\left[K_{N}(d)+\pi_{0}(j) G_{N}(d)+\pi_{0}(a(j))\left(1-G_{N}(d)\right)\right. & \text { if } 1 \leq n \leq N-1, \\
\left.\quad+\beta\left(V(b(j), 1) G_{N}(d)+V(b(a(j)), 1)\left(1-G_{N}(d)\right)\right)\right] & \text { if } \quad n-N .\end{cases}
\end{aligned}
$$

\footnotetext{
${ }^{3}$ A similar set of equations derived intuitively is also discussed by Norman and Shearn (1980).
} 
In order to rewrite the above system in a compact matrix notation we introduce the following conventions. Let $\mathscr{F}$ denote the set of elements $(j, n)$, i.e.,

$$
\mathscr{F}=\{(2,1), \ldots,(J, 1),(1,2), \ldots,(J, 2), \ldots,(1, N), \ldots,(J, N)\}
$$

and let

$$
f:=(f(j, n))_{(j, n) \in \mathscr{F}}=(f(2,1), \ldots, f(J, 1), \ldots, f(1, N), \ldots, f(J, N))
$$

be an arbitrary vector on $\mathscr{F}$, i.e. $f \in \mathbb{R}^{\mathscr{F}}$.

Define

$$
\begin{aligned}
& \boldsymbol{v}:=(V(j, n))_{(j, n) \in \mathscr{F}}, \\
& \boldsymbol{d}:=(D(j, n))_{(j, n) \in \mathscr{F}}, \\
& r(\boldsymbol{f}):=(R(\boldsymbol{f})(j, n))_{(j, n) \in \mathscr{F}}, \quad f \geq 0,
\end{aligned}
$$

with

$$
\begin{aligned}
R & (f)(j, n) \\
& = \begin{cases}K_{n}(f(j, n)), & 1 \leq n \leq N-1, \\
K_{N}(f(j, N))+G_{N}(f(j, N)) \pi_{0}(j)+\pi_{0}(a(j))\left(1-G_{N}(f(j, N))\right) & \text { if } \quad n=N,\end{cases}
\end{aligned}
$$

and $D(j, n)$ given by (18) and (19).

Moreover, let $Q(f)$ be the Markov matrix of the underlying Markov chain if the policy holder uses a threshold policy $f \in \mathbb{R}^{\mathscr{F}}, f \geq 0$. Hence

$$
Q(\boldsymbol{f})=\left(\begin{array}{cccc}
\mathbf{0} & Q_{1}(\boldsymbol{f}) & \ldots & \mathbf{0} \\
\vdots & & & \vdots \\
\vdots & & & Q_{N-1}(\boldsymbol{f}) \\
Q_{N}(\boldsymbol{f}) & \ldots & \ldots & \mathbf{0}
\end{array}\right)
$$

This matrix consists of submatrices $Q_{n}(f)=\left(q_{n}(f)((j, n),(k, m))\right), 1 \leq n \leq N$, satisfying

$$
q_{n}(f)((j, n),(k, m))=\left\{\begin{array}{lll}
G_{n}(f(j, n)) & \text { if } \quad k=j ; m=n+1 ; 1 \leq n \leq N-1, \\
G_{N}(f(j, n)) & \text { if } \quad k=b(j) ; m=1 ; n=N, \\
1-G_{n}(f(j, n)) & \text { if } \quad k=a(j) ; m=n+1 ; 1 \leq n \leq N-1, \\
1-G_{n}(f(j, n)) & \text { if } k=b(a(j)) ; m=1 ; n=N, \\
0 & \text { elsewhere. }
\end{array}\right.
$$

Finally, we introduce the set of operators $L(f), f \geq 0$ and $U: \mathbb{R}^{S t} \rightarrow \mathbb{R}^{3 / 4}$ defined by

$$
L(f) w=r(f)+\beta Q(f) w
$$

and

$$
U_{w}=\min _{\boldsymbol{f} \geq 0} L(f) w,
$$

where the minimization is taken component-wise.

It is not difficult to verify that the pair of relationships (20), (21) and (22), (23) can be written as

$$
v=L(d) v
$$

and

$$
v=U v
$$

Also, by Lemma A.1 we obtain, for every $\boldsymbol{w} \in \mathbb{R}^{\mathscr{F}}$,

$$
U \boldsymbol{w}=L\left(\boldsymbol{f}_{\boldsymbol{w}}\right) \boldsymbol{w} \text { where } \boldsymbol{f}_{\boldsymbol{w}}:=\left(f_{\mathfrak{w}}(j, n)\right)_{(j, n) \in \mathscr{F}}
$$


with

$$
f_{w}(j, n):= \begin{cases}\max (\beta(w(a(j), n+1)-w(j, n+1)), 0) & \text { if } \quad 1 \leq n \leq N-1, \\ \max \left(\pi_{0}(a(j))-\pi_{0}(j)+\beta(w(b(a(j)), 1)-w(b(j), 1)), 0\right) & \text { if } \quad n=N .\end{cases}
$$

Hence using well-known arguments from the theory of Markov Decision Processes [cf. Ross (1970)] we obtain the next result. Before discussing this result we introduce the so-called maxnorm $\|\bullet\|$ on $\mathbb{R}^{\mathscr{F}}$, i.e., $\|\boldsymbol{w}\|:=\max _{(j, n)}|w(j, n)|$.

Lemma 3.1. (a). The operator $U: \mathbb{R}^{\mathscr{F}} \rightarrow \mathbb{R}^{\mathscr{F}}$ is a $\beta$-contraction mapping, i.,e. $\left\|U \boldsymbol{w}_{1}-U \boldsymbol{w}_{2}\right\| \leq \beta\left\|\boldsymbol{w}_{1}-\boldsymbol{w}_{2}\right\|$ for every bounded $w_{1}, w_{2} \in \mathbb{R}^{\mathscr{F}}$.

(b) The equation $U \boldsymbol{w}=\boldsymbol{w}$ has a unique solution and this solution equals $\boldsymbol{v}$..

Proof. Part (a) can be proved by using relationship (28) and Theorem 6.5 of Ross (1970). The result in (b) is an immediate consequence of (a) and (27).

By Lemma 3.1 and the result discussed in the appendix, it is clear that $\lim _{n \rightarrow \infty}\left\|v_{n}-v\right\|=0$ where $\boldsymbol{v}_{n}:=U \boldsymbol{v}_{n-1}=L\left(\boldsymbol{f}_{\boldsymbol{v}_{n-1}}\right) \boldsymbol{v}_{n-1}, n \geq 1$ and $\boldsymbol{v}_{0}$ is some bounded vector belonging to $\mathbb{R}^{\mathbb{F}}$.

The method of successive approximations is based upon this observation. However, before introducing this method and the corresponding algorithm, we have to define a stopping rule. The form of this stopping rule can be derived from the following well-known result.

Lemma 3.2. Suppose $\boldsymbol{v}_{n}=\boldsymbol{U} \boldsymbol{v}_{n-1}, n \geq 1$ and $\boldsymbol{v}_{0}$ is an arbitrary bounded vector on $\mathscr{F}$. If $\mathrm{e}$ denotes the vector on $\mathscr{F}$ with all components equal to 1 , then for every $n \geq 1$ the next inequality holds:

$$
v_{n}+\frac{\beta}{1-\beta} \min _{(j, n)}\left(\left(v_{n}-v_{n-1}\right)(j, n)\right) \boldsymbol{e} \leq \boldsymbol{v} \leq \boldsymbol{v}_{n}+\frac{\beta}{1-\beta} \max _{(j, n)}\left(\left(v_{n}-v_{n-1}\right)(j, n)\right) \boldsymbol{e} .
$$

Moreover, the lowerbound (upperbound) converges monotonically increasing (decreasing) to $v$ as $n \rightarrow \infty$.

Proof. In order to prove these so-called McQueen bounds we use relation (26) and copy the proof of the above result for Markov Decision Processes with finite action spaces [cf. Hendrikx, Van Nunen and Wessels (1984)].

Defining $\operatorname{span}(v):=\max _{(j, n)}(v(j, n))-\min _{(j, n)}(v(j, n))$ we are now able to state the method of successive approximations.

\section{Algorithm}

Step 1. Choose $v_{0}$ some arbitrary bounded vector on $\mathscr{F}$.

Step 2. Compute, for $n \geq 1$,

$$
\boldsymbol{v}_{n}=U \boldsymbol{v}_{n-1}=L\left(f_{\boldsymbol{v}_{n-1}}\right) \boldsymbol{v}_{n-1} .
$$

Step 3. If span $\left(v_{n}-v_{n-1}\right)<\epsilon$ for some given $\epsilon>0$, stop.

Otherwise, return to step 2 with $n:=n+1$.

$$
\begin{aligned}
\text { Output: } \quad \hat{\boldsymbol{v}}: & =v_{n}+\frac{1}{2} \frac{\beta}{1-\beta}\left(\max _{(j, n)}\left(\left(v_{n}-v_{n-1}\right)(j, n)\right)+\min _{(j, n)}\left(\left(v_{n}-v_{n-1}\right)(j, n)\right)\right), \\
\hat{\boldsymbol{d}} & :=f_{\hat{v}} .
\end{aligned}
$$

By Lemma 3.2 and the definition of $\hat{\boldsymbol{d}}$ is is easy to derive the next result. 
Theorem 3.3. After completion of the above algorithm the following holds:

$$
\|\hat{d}-d\| \leq \beta^{2} \varepsilon /(1-\beta) .
$$

Proof. By Lemma 3.2 we easily obtain

$$
\|\hat{\boldsymbol{v}}-\boldsymbol{v}\| \leq \frac{1}{2} \cdot \frac{\beta}{1-\beta} \operatorname{span}\left(\boldsymbol{v}_{n}-\boldsymbol{v}_{n-1}\right)
$$

Moreover, by the definition of $\hat{\boldsymbol{d}}$ and (18) and (19), it is also not difficult to verify that

$$
\|\hat{\boldsymbol{d}}-\boldsymbol{d}\| \leq 2 \beta\|\hat{\boldsymbol{v}}-\boldsymbol{v}\|
$$

and hence combining the above inequalities yields the desired result.

The proof of Theorem 3.3 concludes this section. In the next section we will discuss some sensitivity results.

\section{Sensitivity analysis}

In this section we will prove some sensitivity results for both models discussed. In particular we will compare the optimal value-functions associated with the vectors of input parameters $\eta_{1}=\left(\beta, \boldsymbol{p}_{1}^{\mathrm{T}}, \boldsymbol{F}_{1}^{\mathrm{T}}\right)^{\mathrm{T}}$ and $\eta_{2}=\left(\beta, \boldsymbol{p}_{2}^{\mathrm{T}}, \boldsymbol{F}_{2}^{\mathrm{T}}\right)^{\mathrm{T}}$ whenever $\boldsymbol{p}_{1} \neq \boldsymbol{p}_{2}$ or $\boldsymbol{F}_{1} \neq \boldsymbol{F}_{2}$ with $\boldsymbol{p}_{i}(i=1,2)$ the vector of probabilities of accident occurrences in subperiods $n=1, \ldots, N$ and $\boldsymbol{F}_{i}(i=1,2)$ the vector of damage distribution in subperiods $n=1, \ldots, N$.

By giving an upper bound on the difference of these value-functions in the maxnorm $\|\bullet\|$, we can easily obtain a similar type of result for the difference of the vectors of optimal critical claim sizes. It turns out that the models discussed are robust and this implies that the optimal critical claim sizes obtained after an unbiased estimation of the input parameters are close to the real optimal critical claim sizes if the number of observations will become large. Starting the analysis of the above problem for the finite horizon model we introduce the following notations. Let $\mathscr{F}_{n}$ denote the set of elements of $\mathscr{F}$ whose second component is $n$, i.e., $\mathscr{F}_{n}=(\ldots,(3, n), \ldots,(J, n)\}$ and define for the input vector $\eta_{m}, m=1,2$,

$$
\boldsymbol{v}_{m, N i-n}:=\left(V_{i}(j, n)\right)_{(j, n) \in \mathscr{F}_{n}}^{\mathrm{T}}, \quad r_{m, n}(f):=(R(f)(j, n))_{(j, n) \in \mathscr{F}_{n}}^{\mathrm{T}},
$$

where $R(f)(j, n)$ and $V_{i}(j, n)$ are introduced in (24) and (11).

Moreover, $L_{m, n}(f)$ and $U_{m, n}$ denote the operators $L(f)$, resp. $U$ restricted to $\mathbb{R}^{\mathscr{F}_{n+1}}$ (if $1 \leq n \leq N-1$ ) or $\mathbb{R}_{1}^{\mathscr{F}}$ (if $n=N$ ) for the same input vector $\eta_{m}$, i.e.,

$$
L_{m, n}(f) w:=r_{m, n}(f)+\beta Q_{m, n}(f) w \quad \text { and } \quad U_{m, n} w:=\min _{\boldsymbol{f}} L_{m, n}(f) w
$$

for all $w \in \mathbb{R}^{\mathscr{F}_{n+1}}$ (if $1 \leq n \leq N-1$ ) or $w \in \mathbb{R}^{\mathscr{F}_{1}}$ (if $n=N$ ). By Lemma A.1 it is clear that the relationships (11) and (12) can be written in the following compact matrix notation:

$$
v_{m, N i-n}=U_{m, n} v_{m, N i-n-1}, \quad 2 \leq i \leq T, \quad 1 \leq n \leq N, \quad m=1,2 .
$$

This relationship will be the starting point of the proof of the next result.

Theorem 4.1 ( finite horizon).

(a) For every pair of vectors $\eta_{m}=\left(\beta, \boldsymbol{p}_{m}^{T}, \boldsymbol{F}_{m}^{\mathbf{T}}\right)^{T}, m=1,2$, satisfying $\boldsymbol{p}_{1} \neq \boldsymbol{p}_{2}$ the following inequality holds:

$$
\left\|\boldsymbol{v}_{1, N T-1}-\boldsymbol{v}_{2, N T-1}\right\| \leq\left\|\boldsymbol{p}_{1}-\boldsymbol{p}_{2}\right\| \frac{1-\beta^{N(T-1)}}{1-\beta^{N}} \sum_{k=0}^{N-1} \beta^{k} \mathbb{E}\left(\underline{X}_{k+1}\right) .
$$


(b) For every pair of vectors $\eta_{m}=\left(\beta, \boldsymbol{p}^{\mathrm{T}}, \boldsymbol{F}_{m}^{\mathrm{T}}\right)^{\mathrm{T}}, m=1,2$, satisfying $\boldsymbol{F}_{1} \neq \boldsymbol{F}_{2}$ the following inequality holds:

$$
\left\|v_{1, N T-1}-v_{2, N T-1}\right\| \leq \frac{1-\beta^{N(T-1)}}{1-\beta^{N}}\|p\| \sum_{k=0}^{N-1} \beta^{k} L\left(F_{k+1,1}, F_{k+1,2}\right)
$$

where $F_{n . m}(m=1,2)$ denotes the nth component of the vector $\boldsymbol{F}_{m}$ and

$$
L\left(F_{n, 1}, F_{n, 2}\right):=\int_{0}^{\infty}\left|F_{n, 1}(z)-F_{n, 2}(z)\right| \mathrm{d} z .
$$

Proof. Since (a) and (b) can be proved in a similar way we will only consider the proof of (a). By (29) we have, for every $2 \leq i \leq T$ and $1 \leq n \leq N$,

$$
\begin{aligned}
& \left\|\boldsymbol{v}_{1, N_{i}-n}-\boldsymbol{v}_{2, N i-n}\right\|=\mid U_{1, n} \boldsymbol{v}_{1, N i-n-1}-U_{2, n} \boldsymbol{v}_{2, N i-n-1} \| \\
& \leq\left\|U_{1, n} \boldsymbol{v}_{1, N i-n-1}-U_{1, n} \boldsymbol{v}_{2, N i-n-1}\right\|+\left\|U_{1, n} \boldsymbol{v}_{2, N i-n-1}-U_{2, n} \boldsymbol{v}_{2, N i-n-1}\right\| .
\end{aligned}
$$

Since $U_{1, n}$ is a $\beta$-contraction mapping for every $1 \leq n \leq N$ clearly the first term in the above inequality is bounded by $\beta\left\|v_{1, N i-n-1}-v_{2, N i-n-1}\right\|$.

Moreover, by Lemma A.1 we obtain

$$
U_{m, n} \boldsymbol{v}_{2, N i-n-1}=L_{m, n}\left(\boldsymbol{d}_{2, i}\right) \boldsymbol{v}_{2, N i-n-1}
$$

for every $m=1,2$ and $\boldsymbol{d}_{2, i}:=\left\{D_{i}(j, n)\right)_{(j, n) \in \mathscr{F}}$ with $D_{i}(j, n)$ given by (7) and (8) using the input vector $\eta_{2}$. This yields

$$
\left\|U_{1, n} \boldsymbol{v}_{2, N i-n-1}-U_{2, n} \boldsymbol{v}_{2, N i-n-1}\right\|=\left\|L_{1, n}\left(\boldsymbol{d}_{2, i}\right) \boldsymbol{v}_{2, N i-n-1}-L_{2, n}\left(\boldsymbol{d}_{2, i}\right) \boldsymbol{v}_{2, N i-n-1}\right\|
$$

and hence after some calculations, using the definition of $L_{m, n}\left(\boldsymbol{d}_{2, i}\right)(m=1,2)$ we obtain

$$
\begin{aligned}
\left\|U_{1, n} \boldsymbol{v}_{2, N i-n-1}-U_{2, n} \boldsymbol{v}_{2, N i-n-1}\right\| & =\max _{(j, n)}\left|p_{n, 1}-p_{n, 2}\right| \int_{0}^{D_{i}(j, n)}\left(1-F_{n}(x)\right) \mathrm{d} x \\
& \leq\left\|\boldsymbol{p}_{1}-\boldsymbol{p}_{2}\right\| \mathbb{E} \underline{X}_{n},
\end{aligned}
$$

where $p_{n, m}$ denotes the $n$th component of the vector $\boldsymbol{p}_{m}, m=1,2$.

Combining the above inequalities yields for every $2 \leq i \leq T$ and $1 \leq n \leq N$ that

$$
\left\|\boldsymbol{v}_{1, N i-n}-\boldsymbol{v}_{2, N i-n}\right\| \leq \beta\left\|\boldsymbol{v}_{1, N i-n-1}-\boldsymbol{v}_{2, N i-n-1}\right\|+\left\|p_{1}-p_{2}\right\| \mathbb{E} \underline{X}_{n} .
$$

Since the value-functions $v_{1, n}$ and $v_{2, n}, 1 \leq n \leq N-1$ are equal to the vector consisting of zeros, we obtain by iterating relationship (30) that

$$
\begin{aligned}
\left\|\boldsymbol{v}_{1, N T-1}-\boldsymbol{v}_{2, N T-1}\right\| & \leq \beta\left\|\boldsymbol{v}_{1, N T-2}-\boldsymbol{v}_{2, N T-2}\right\|+\left\|\boldsymbol{p}_{1}-\boldsymbol{p}_{2}\right\| \mathbb{E} \underline{X}_{1} \\
& \leq \ldots \leq \sum_{j=0}^{T-2} \beta^{N j} \sum_{k=0}^{N-1} \beta^{k} \mathbb{E}\left(\underline{X}_{k+1}\right)\left\|\boldsymbol{p}_{1}-\boldsymbol{p}_{2}\right\|+\beta^{N(T-1)}\left\|\boldsymbol{v}_{1, N-1}-\boldsymbol{v}_{2, N-1}\right\| \\
& =\frac{\left.1-\beta^{N(T} 1\right)}{1-\beta^{N}} \sum_{k=0}^{N-1} \beta^{k} \mathbb{E}\left(\underline{X}_{k+1}\right)
\end{aligned}
$$

and hence the desired result is proved.

By the observation that the infinite horizon model can be approximated by a sequence of finite horizon models (let $T \rightarrow \infty$ ) we immediately obtain he following result.

Theorem 4.2. (Infinite horizon).

(a) For every pair of vectors $\eta_{m}=\left(\beta, \boldsymbol{p}_{m}^{\mathrm{T}}, \boldsymbol{F}^{\mathrm{T}}\right)^{\mathrm{T}}, m=1,2$, satisfying $\boldsymbol{p}_{1} \neq \boldsymbol{p}_{2}$ the following inequality holds:

$$
\left\|v_{1}-v_{2}\right\| \leq\left\|p_{1}-p_{2}\right\|\left(1-\beta^{N}\right)^{-1} \sum_{k=0}^{N-1} \beta^{k} \mathbb{E}\left(\underline{X}_{k+1}\right) .
$$


(b) For every pair of vectors $\eta_{m}=\left(\beta, \boldsymbol{p}^{\mathrm{T}}, \boldsymbol{F}_{m}^{\mathrm{T}}\right)^{\mathrm{T}}, m=1,2$, satisfying $\boldsymbol{F}_{1} \neq \boldsymbol{F}_{2}$ the following inequality holds:

$$
\left\|\boldsymbol{v}_{1}-\boldsymbol{v}_{2}\right\| \leq\|\boldsymbol{p}\|\left(1-\beta^{N}\right)^{-1} \sum_{k=0}^{N-1} \beta^{k} L\left(F_{k+1,1}, F_{k+1,2}\right) .
$$

Proof. Apply Theorem 4.1 and let $T \rightarrow \infty$.

By the above theorems it is not difficult to obtain similar types of results for the optimal critical claim sizes associated with the input parameters $\eta_{m}(m=1,2)$ using $\left\|\boldsymbol{d}_{1}-\boldsymbol{d}_{2}\right\| \leq 2 \beta\left\|\boldsymbol{v}_{1}-\boldsymbol{v}_{2}\right\|$. This concludes the section on sensitivity. In the next section we will discuss some computations.

\section{Results}

The model presented in Section 3 will be applied to the bonus-malus system given in Table 1 . We divide an insurance year in $N$ equal periods, for instance weeks or months and we assume that the probability $p_{n}$ to have an accident during period $n$ equals $\lambda / N$. Usually the number of accidents during a year is assumed to be Poisson-distributed, but the above choice is only slightly different for relevant values of $\lambda$. We assume the amount of damage $\underline{Y}_{n}$ to be lognormally distributed with parameters $\mu$ and $\sigma^{2}$. For reasons of simplicity we assume the parameters $\lambda, \mu$ and $\sigma^{2}$ to be independent of $n$, hence we assume that the parameter values are constant throughout the entire duration of the decision process.

For a third-party liability insurance a value of $\lambda=0.1$ accidents per year is reasonable. The values of $\mu$ and $\sigma^{2}$ are assumed to be $\mu=6.98849$ and $\sigma^{2}=1.0213$, which corresponds to a mathematical expectation equal to Dfl. 1800 and a modus equal to Dfl. 389.

The basic premium is equated to Dfl. 1000, and the annual interest rate to 5 percent. Therefore, the annual discount rate equals $\beta_{0}=0.95238$ and the discount rate per period equals $\beta=0.99594$ for $N=12$. As stop criterion for the infinite horizon model we take $\epsilon=0.00001$. Substituting the values of $\beta$ and $\epsilon$ in the formula given by Theorem 3.3, we obtain in this case

$$
\|\hat{d}-\boldsymbol{d}\| \leq 0.0024 \text {. }
$$

This corresponds to a relative error for the critical claim sizes which is less than 0.0005 percent. We obtain similar results if we take $N=52$ in combination with $\beta=0.99906$.

Table 4

Increase of the premium when claiming once or twice.

\begin{tabular}{rrr}
\hline$j$ & $\Delta \rho_{0}$ & $\Delta \rho_{1}$ \\
\hline 1 & 0.0 & 0.0 \\
2 & 20.0 & 0.0 \\
3 & 30.0 & 0.0 \\
4 & 40.0 & 0.0 \\
5 & 50.0 & 0.0 \\
6 & 60.0 & 0.0 \\
7 & 40.0 & 20.0 \\
8 & 45.0 & 20.0 \\
9 & 35.0 & 30.0 \\
10 & 30.0 & 40.0 \\
11 & 25.0 & 50.0 \\
12 & 20.0 & 60.0 \\
13 & 17.5 & 45.0 \\
14 & 20.0 & 35.0 \\
15 & 17.5 & 30.0 \\
16 & 20.0 & 30.0 \\
17 & 15.0 & 25.0 \\
\hline
\end{tabular}


Table 5

Optimal critical claim sizes for a horizon of 10 years, in Dfl.

\begin{tabular}{|c|c|c|c|c|c|c|c|c|c|c|c|c|}
\hline \multirow[t]{2}{*}{$j$} & \multicolumn{12}{|l|}{$n$} \\
\hline & 1 & 2 & 3 & 4 & 5 & 6 & 7 & 8 & 9 & 10 & 11 & 12 \\
\hline 1 & & 0 & 0 & 0 & 0 & 0 & $\overline{0}$ & 0 & 0 & 0 & 0 & 0 \\
\hline 2 & 586 & 593 & 600 & 607 & 614 & 621 & 628 & 635 & 642 & 650 & 657 & 665 \\
\hline 3 & 1075 & 1086 & 1098 & 1109 & 1121 & 1132 & 1144 & 1156 & 1168 & 1180 & 1192 & 1204 \\
\hline 4 & 1536 & 1551 & 1566 & 1581 & 1596 & 1611 & 1627 & 1642 & 1657 & 1673 & 1689 & 1705 \\
\hline 5 & 1959 & 1976 & 1994 & 2012 & 2030 & 2048 & 2066 & 2084 & 2103 & 2121 & 2140 & 2159 \\
\hline 6 & & 2338 & 2358 & 2378 & 2399 & 2419 & 2439 & 2460 & 2480 & 2501 & 2522 & 2543 \\
\hline 7 & 1746 & 1758 & 1770 & 1782 & 1794 & 1806 & 1818 & 1830 & 1842 & 1854 & 1866 & 1878 \\
\hline 8 & & 2038 & 2052 & 2065 & 2079 & 2093 & 2107 & 2121 & 2134 & 2148 & 2162 & 2177 \\
\hline 9 & 1549 & 1557 & 1565 & 1573 & 1581 & 1589 & 1597 & 1605 & 1613 & 1621 & 1629 & 1637 \\
\hline 10 & 1341 & 1346 & 1351 & 1356 & 1361 & 1365 & 1370 & 1375 & 1380 & 1384 & 1389 & 1394 \\
\hline 11 & 1130 & 1132 & 1134 & 1136 & 1138 & 1140 & 1142 & 1143 & 1145 & 1147 & 1149 & 1151 \\
\hline 12 & 934 & 934 & 933 & 933 & 933 & 932 & 931 & 931 & 930 & 929 & 929 & 928 \\
\hline 13 & 755 & 754 & 753 & 751 & 750 & 748 & 746 & 745 & 743 & 741 & 740 & 738 \\
\hline 14 & 827 & 827 & 828 & 828 & 829 & 829 & 830 & 830 & 830 & 831 & 831 & 831 \\
\hline 15 & 642 & 641 & 640 & 640 & 639 & 639 & 638 & 637 & 636 & 636 & 635 & 634 \\
\hline 16 & 677 & 677 & 677 & 676 & 676 & 676 & 675 & 675 & 675 & 674 & 674 & 674 \\
\hline 17 & 480 & 478 & 477 & 475 & 474 & 472 & 471 & 469 & 467 & 466 & 464 & 462 \\
\hline
\end{tabular}

To interpret the results it is useful to examine the influence of the filing of a claim on the premium to be paid by the policy holder, where we denote the premium as a percentage of the basic premium. Let

$$
\Delta \rho_{0}(j)=\rho_{1}(j)-\rho_{0}(j), \quad \Delta \rho_{1}(j)=\rho_{1}(a(j))-\rho_{1}(j),
$$

where the definitions of $\rho_{0}(j)$ and $\rho_{1}(j)$ are given in the footnote under Table 2.

The value of $\Delta \rho_{0}(j)$ equals the increase of the premium when one additional claim, and $\Delta \rho_{1}(j)$ equals the additional increase of the premium when a second additional claim is filed during the current year. Table 4 contains the values of $\Delta \rho_{0}(j)$ and $\Delta \rho_{1}(j)$ for each $j, j=1, \ldots, 17$, derived from Table 2 .

Table 6

Optimal critical claim sizes for a horizon of 25 years, in Dfl.

\begin{tabular}{|c|c|c|c|c|c|c|c|c|c|c|c|c|}
\hline \multirow[t]{2}{*}{$j$} & \multicolumn{12}{|l|}{$n$} \\
\hline & 1 & 2 & 3 & 4 & 5 & 6 & 7 & 8 & 9 & 10 & 11 & 12 \\
\hline 1 & & 0 & 0 & 0 & 0 & 0 & 0 & 0 & 0 & 0 & 0 & 0 \\
\hline 2 & 668 & 675 & 683 & 691 & 699 & 707 & 715 & 723 & 731 & 739 & 747 & 756 \\
\hline 3 & 1228 & 1241 & 1253 & 1266 & 1279 & 1292 & 1305 & 1318 & 1331 & 1344 & 1357 & 1371 \\
\hline 4 & 1748 & 1764 & 1780 & 1797 & 1813 & 1830 & 1847 & 1863 & 1880 & 1898 & 1915 & 1932 \\
\hline 5 & 2212 & 2231 & 2250 & 2270 & 2289 & 2309 & 2328 & 2348 & 2368 & 2388 & 2409 & 2429 \\
\hline 6 & & 2617 & 2639 & 2660 & 2682 & 2704 & 2726 & 2748 & 2771 & 2793 & 2816 & 2838 \\
\hline 7 & 1942 & 1955 & 1967 & 1980 & 1993 & 2005 & 2018 & 2031 & 2044 & 2057 & 2070 & 2083 \\
\hline 8 & & 2252 & 2267 & 2281 & 2296 & 2310 & 2325 & 2340 & 2355 & 2369 & 2384 & 2399 \\
\hline 9 & 1691 & 1700 & 1708 & 1716 & 1725 & 1733 & 1741 & 1750 & 1758 & 1767 & 1775 & 1784 \\
\hline 10 & 1439 & 1444 & 1449 & 1453 & 1458 & 1463 & 1468 & 1473 & 1478 & 1483 & 1488 & 1493 \\
\hline 11 & 1195 & 1197 & 1199 & 1201 & 1203 & 1205 & 1206 & 1208 & 1210 & 1212 & 1214 & 1216 \\
\hline 12 & 980 & 980 & 979 & 979 & 978 & 978 & 977 & 976 & 976 & 975 & 974 & 973 \\
\hline 13 & 787 & 785 & 784 & 782 & 781 & 779 & 777 & 775 & 774 & 772 & 770 & 768 \\
\hline 14 & 860 & 860 & 860 & 861 & 861 & 862 & 862 & 862 & 862 & 863 & 863 & 863 \\
\hline 15 & 662 & 662 & 661 & 660 & 660 & 659 & 658 & 657 & 656 & 656 & 655 & 654 \\
\hline 16 & 698 & 698 & 698 & 697 & 697 & 697 & 696 & 696 & 695 & 695 & 694 & 694 \\
\hline 17 & 492 & 491 & 489 & 488 & 486 & 484 & 483 & 481 & 479 & 477 & 476 & 474 \\
\hline
\end{tabular}


Table 7

Optimal critical claim sizes for an infinite horizon, in Dfl.

\begin{tabular}{|c|c|c|c|c|c|c|c|c|c|c|c|c|}
\hline \multirow[t]{2}{*}{$j$} & \multicolumn{12}{|l|}{$n$} \\
\hline & 1 & 2 & 3 & 4 & 5 & 6 & 7 & 8 & 9 & 10 & 11 & 12 \\
\hline 1 & & 0 & 0 & 0 & 0 & 0 & 0 & 0 & 0 & 0 & 0 & 0 \\
\hline 2 & 668 & 676 & 683 & 691 & 699 & 707 & 715 & 723 & 731 & 739 & 747 & 756 \\
\hline 3 & 1229 & 1241 & 1254 & 1267 & 1279 & 1292 & 1305 & 1318 & 1331 & 1345 & 1358 & 1371 \\
\hline 4 & 1748 & 1764 & 1781 & 1797 & 1814 & 1830 & 1847 & 1864 & 1881 & 1898 & 1915 & 1933 \\
\hline 5 & 2212 & 2232 & 2251 & 2270 & 2290 & 2309 & 2329 & 2349 & 2369 & 2389 & 2409 & 2430 \\
\hline 6 & & 2618 & 2640 & 2661 & 2683 & 2705 & 2727 & 2749 & 2771 & 2794 & 2816 & 2839 \\
\hline 7 & 1942 & 1955 & 1968 & 1980 & 1993 & 2006 & 2019 & 2032 & 2044 & 2057 & 2070 & 2083 \\
\hline 8 & & 2253 & 2267 & 2282 & 2296 & 2311 & 2326 & 2340 & 2355 & 2370 & 2385 & 2400 \\
\hline 9 & 1692 & 1700 & 1708 & 1717 & 1725 & 1733 & 1742 & 1750 & 1759 & 1767 & 1776 & 1784 \\
\hline 10 & 1439 & 1444 & 1449 & 1454 & 1459 & 1464 & 1468 & 1473 & 1478 & 1483 & 1488 & 1493 \\
\hline 11 & 1195 & 1197 & 1199 & 1201 & 1203 & 1205 & 1207 & 1208 & 1210 & 1212 & 1214 & 1216 \\
\hline 12 & 980 & 980 & 980 & 979 & 978 & 978 & 977 & 977 & 976 & 975 & 974 & 973 \\
\hline 13 & 787 & 786 & 784 & 782 & 781 & 779 & 777 & 775 & 774 & 772 & 770 & 768 \\
\hline 14 & 860 & 860 & 861 & 861 & 861 & 862 & 862 & 862 & 862 & 863 & 863 & 863 \\
\hline 15 & 663 & 662 & 661 & 660 & 660 & 659 & 658 & 657 & 656 & 656 & 655 & 654 \\
\hline 16 & 699 & 698 & 698 & 697 & 697 & 697 & 696 & 696 & 695 & 695 & 694 & 694 \\
\hline 17 & 492 & 491 & 489 & 488 & 486 & 484 & 483 & 481 & 479 & 477 & 476 & 474 \\
\hline
\end{tabular}

We compute the optimal critical claim sizes for horizons of 10 years and 25 years and for an infinite horizon, both for $N=12$ and $N=52$. To avoid spatial problems, only the results for $N=12$ are given in Tables 5-7.

We make the following remarks.

(1) From Table 5 it follows that the combinations $(j, m)=(1,1),(6,1)$ and $(8,1)$ cannot occur.

(2) The results obtained for a finite horizon of 25 years (Table 6) differ only little from the results for an infinite horizon (Table 7).

(3) The estimated optimal critical claim sizes show a pattern similar to $\Delta \rho_{0}$ for any fixed value of $n$. That is, when the premium increases relatively little $\left(\Delta \rho_{U}\right.$ low $)$, then $\hat{d}$ will be relatively low, and the other way round.

(4) The values of $\hat{\boldsymbol{d}}$ increases with $n$ for $n=1, \ldots, 11$, and 14, decreases with $n$ for $n=12,13$ and 15, 16, 17. This pattern changes slightly during the last years of the insurance, but since it originates from differences between expected costs in various states it is difficult to explain this particular pattern conclusively.

(5) The longer the process continues before it ends, the higher the values of $\hat{\boldsymbol{d}}$ are. For the higher the number of years before the process ends, the more important it is for a policy holder to be in a high bonus-malus class, and consequently the higher $\boldsymbol{d}$ is.

(6) The values of $\hat{\boldsymbol{d}}$ for $N=52$ are almost linear interpolations between the values for $N=12$. Therefore, and for spatial reasons, we do not present these values.

\section{Appendix}

In this appendix we prove the next result.

Lemma A.1. Let $\mathscr{F}$ denote the set of elements $(j, n)$ and $w: \mathscr{F} \rightarrow \mathbb{R}$ is an arbitrary vector on $\mathscr{F}$. If

$$
f_{w}:=\left(f_{w}(j, n)\right)_{(j, n) \in \mathscr{T}}
$$

with

$$
f_{w}(j, n):= \begin{cases}\max (\beta(w(a(j), n+1)-w(j, n+1)), 0) & \text { if } \quad 1 \leq n \leq N-1, \\ \max \left(\pi_{0}(a(j))-\pi_{0}(j)+\beta(w(b(a(j)), 1)-w(b(j), 1)), 0\right) & \text { if } \quad n=N,\end{cases}
$$


then for every threshold policy $f \in \mathbb{R}^{\mathscr{F}}, f \geq 0$ and $\eta=\left(\beta, p^{\mathrm{T}}, \boldsymbol{F}^{\mathrm{T}}\right)^{\mathrm{T}}$ we obtain

$$
L\left(\boldsymbol{f}_{w}\right) \boldsymbol{w} \leq L(\boldsymbol{f}) \boldsymbol{w} .
$$

Proof. By definition [cf. (22)] it holds true that

$$
L(f) w=r(f)+\beta Q(f) w
$$

for every threshold policy $f$.

In scalar notation this reads

$$
\begin{aligned}
& L(\boldsymbol{f}) \boldsymbol{w}(j, n) \\
& = \begin{cases}\int_{0^{-}}^{f(j, n)}(x+\beta w(j, n+1)) \mathrm{d} G_{n}(x)+\int_{f(j, n)^{+}}^{\infty} \beta w(a(j), n+1) \mathrm{d} G_{n}(x) & \text { if } 1 \leq n \leq N-1, \\
\int_{0}^{f(j, n)}\left(x+\pi_{0}(j)+\beta w(b(j), 1)\right) \mathrm{d} G_{N}(x)+\int_{f(j, n)}^{\infty}\left(\pi_{0}(a(j))+\beta w(b(a(j)), 1)\right) \mathrm{d} G_{N}(x)\end{cases}
\end{aligned}
$$

We now consider the following two cases for every $(j, n)$.

(i) $f_{w}(j, n)=0$. By the definition of $G_{n}$ relation (A.1) reduces to

$$
L(f) w(j, n)= \begin{cases}\beta\left(\left(1-p_{n}\right) w(j, n+1)+p_{n} w(a(j), n+1)\right) & \text { if } \quad 1 \leq n \leq N-1, \\ p_{N} \pi_{0}(a(j))+\left(1-p_{N}\right) \pi_{0}(j) & \\ \quad+\beta\left(\left(\left(1-p_{N}\right) w(b(j), 1)+p_{N} w(b(a(j)), 1)\right)\right. & \text { if } \quad n=N .\end{cases}
$$

Since $f_{w}(j, n)=0$ we obtain

$$
w(a(j), n+1) \leq w(j, n+1) \quad \text { if } \quad 1 \leq n \leq N-1
$$

or

$$
\pi_{0}(a(j))+\beta w(b(a(j)), 1) \leq \pi_{0}(j)+\beta w(b(j), 1) \quad \text { if } \quad n=N .
$$

Hence by (A.1) up to (A.4)

$$
L\left(f_{w}\right) w(j, n) \leq L(f) w(j, n)
$$

for every $f \geq 0$.

(ii) $f_{w}(j, n)>0$. By relation (A.1) it follows immediately that

$$
\begin{aligned}
& L\left(\boldsymbol{f}_{\boldsymbol{w}}\right) \boldsymbol{w}(j, n)-L(f) w(j, n) \\
& \quad= \begin{cases}\int_{f(j, n)}^{f_{w}(j . n)}[x-\beta(w(a(j), n+1)-w(j, n+1))] \mathrm{d} G_{n}(x) & \text { if } 1 \leq n \leq N-1, \\
\int_{f(j, n)}^{f_{w}(j, n)}\left[x-\left(\pi_{0}(a(j))-\pi_{0}(j)\right)-\beta(w(b(a(j)), 1)-w(b(j), 1))\right] \mathrm{d} G_{n}(x) & \text { if } n=N .\end{cases}
\end{aligned}
$$

Since $f_{\boldsymbol{w}}(j, n)>0$ we obtain

$$
f_{w^{\prime}}(j, n)= \begin{cases}\beta(w(a(j), n+1)-w(j, n+1)) & \text { if } 1 \leq n \leq N-1, \\ \pi_{0}(a(j))-\pi_{0}(j)+\beta(w(b(a(j), 1)-w(b(j)), 1)) & \text { if } \quad n=N\end{cases}
$$

and this implies, by (A.5)

$$
L\left(f_{w}\right) \boldsymbol{w}(j, n) \leq L(f) \boldsymbol{w}(j, n) .
$$




\section{References}

Alting von Gcusau, B.J.J. (1968). The influence of a no-claim-discount-system on the behaviour of the insured clientele of an insurance company: A first approach. University of Amsterdam, Amsterdam.

Bertsekas, D.P. (1976). Dynamic Programming and Stochastic Control. Academic Press, New York.

De Leve, G. and P.J. Weeda (1968). Driving with Markov-programming. The ASTIN Bulletin, no. 5, 62-86,

De Pril, N. (1978). The efficiency of a bonus-malus system. The ASTIN Bulletin, no. 10, 59-72.

De Pril, N. (1979). Optimal claim decisions for a bonus-malus system: A continuous approach. The ASTIN Bulletin, no. 10, 215-222.

Derron, M. (1965). A theoretical study of the no-claim bonus problem. The ASTIN Bulletin, no. 3, 62-74.

Grenander, U. (1957). Some remarks on bonus systems in automobile insurance. Skandinavisk Aktuarietidskrift 40, $180-198$.

Gürtler, M. (1960). Das subjective Risico in der Motorfahrzeugversicherung. Zeitschrift für die Gesamte Versicherungs-Wissenschaft, no. 49, 203-223.

Gürtler, M. (1961). Der Bonus als Mittel zur Erfassung des subjectiven Risicos. Zeitschrift für die Gesamte Versicherungs-Wissenschaft, no. 1, 1-47.

Gürtler, M. (1962). Der optimale Bonus. Zeitschrift für die Gesamte Versicherungs-Wissenschaft, no. 3, 4-51.

Haehling von Lanzenauer, C. (1969). Entscheidungsregeln für das optimale Verhalten in der Kraftfahrtversicherung. Unternemensforschung 13, 193-204.

Haehling von Lanzenauer, C. (1972a). Decision problems in the Canadian automobile insurance systems. Journal of Risk and Insurance 39, 79-92.

Haehling von Lanzenauer, C. (1972b). The effects of the insurees' decisions on the insurer's profit. Proceedings in Operations Research, Physica Verlag, Heidelberg, 399421.

Haehling von Lanzenauer, C. (1974). Optimal claim decisions by policyholders in automobile insurance with merit-rating structures. Operations Research 22, 979-990.

Haehling von Lanzenauer, C. and W.N. Lundberg (1973). The propensity to cause accidents. The ASTIN Bulletin, no. 7, $154-164$.

Hastings, N.A.J. (1976). Optimal claiming on vehicle insurance. Operational Research Quarterly 27, 805-813.

Hendrikx, M., J. van Nunen and J. Wessels (1984). On iterative optimization of structured Markov decision processes with discounted rewards. Math. Operationsforschung und Statistik, Ser. Optimization, 15, 439-459.

Kolderman, J. and A. Volgenant (1985). Optimal claiming in an automobile insurance system with bonus-malus structure. Journal of the Operational Research Society 36, 239-247.

Lemaire, J. (1976). Driver versus company, optimal behaviour of the policy holder. Scandinavian Actuarial Journal 59, $209-219$.

Lemaire, J. (1977). La soif du bonus. The ASTIN Bulletin, no. 9, 181-190.

Lemaire, J. (1985). Automobile Insurance, Actuarial Models. Kluwer-Nijhoff, Boston, MA/Dordrecht/Lancaster.

Loimoranta, K. (1972). Some asymptotic properties of bonus systems, The ASTIN Bulletin, no. 6, 233-245.

Martin-Löf, A. (1973). A method for finding the optimal decision rule for a policy holder of an insurance with a bonus system. Scandinavian Actuarial Journal 56, 23-29.

Menist, M. and A. Volgenant (1986). Optimaal claimgedrag bij autoverzekeringen voor eindige periode, Het Verzekeringsarchief 63, $61-76$.

Norman, J.M. and D.C.S. Shearn (1980). Optimal claiming on vehicle insurance revisited. Journal of the Operational Research Society $31,181-186$

Philipson, C. (1960). The Swedish system of bonus. AUSTIN Bulletin, no. 1, 134-141.

Ross, S.M. (1970). Applied probability models with optimization applications. Holden-Day, San Francisco, CA.

Ross, S.M. (1983). Introduction to stochastic dynamic programming. Academic Press, New York.

Tijms, H.C. (1986). Siochastic modelling and anulysis: A computational approach. Wiley, New York.

Van der Wal, J. and J. Wessels (1985). Markov decision processes. Statistica Neerlandica 39, 219-233.

Vepsäläinen, S. (1972). Applications to a theory of bonus systems. The ASTIN Bulletin, no. 6, 212-221.

Weeda, J. (1975). Technical aspects of the iterative solution of the automobile insurance problem. Report BN $27 / 75$ Mathematical Centre, Amsterdam.

Welten, C.P. (1969). The unearned no claim bonus. The ASTIN Bulletin, no. 5, 25-32. 